\title{
Spin-coating process evolution and reproducibility for power-law fluids
}

\author{
P. L. G. Jardim, ${ }^{1, *}$ A. F. Michels, ${ }^{2}$ and F. Horowitz ${ }^{1}$ \\ 'Instituto de Física-Universidade Federal do Rio Grande do Sul (UFRGS), Campus do Vale, \\ CP15051, CEP 91501-970 Porto Alegre, RS, Brazil \\ ${ }^{2}$ Centro de Ciências Exatas e Tecnologia, Universidade de Caxias do Sul, CEP 95070-560 Caxias do Sul, RS, Brazil \\ *Corresponding author: pedro.lovato@ufrgs.br
}

Received 12 November 2013; revised 14 February 2014; accepted 14 February 2014; posted 14 February 2014 (Doc. ID 201196); published 19 March 2014

\begin{abstract}
A distinct development of an exact analytical solution for power-law fluids during the spin-coating process is presented for temporal and spatial thickness evolution, after steady state conditions are attained. This solution leads to the definition of a characteristic time, related to the memory of the initial thickness profile. Previously obtained experimental data, for several rotation speeds and carboxymetilcellulose concentrations in water, are quantitatively analyzed through the evaluation of their characteristic times and compared with theoretical predictions, thus allowing better understanding of thickness profile evolution and of process reproducibility. (C) 2014 Optical Society of America

OCIS codes: (120.3180) Interferometry; (160.5470) Polymers; (240.0310) Thin films; (260.3160)

Interference; (310.1620) Interference coatings; (310.6860) Thin films, optical properties.

http://dx.doi.org/10.1364/AO.53.001820
\end{abstract}

\section{Introduction}

Reproducibility is a remarkable property in the spin-coating process, well known since its early use for painting plane surfaces [1], and is critical to optical and microelectronics device fabrication. The theoretical models for flow description during spin coating must, therefore, be able to demonstrate this characteristic aspect at some point.

The Emslie et al. [2] pioneering model, for Newtonian flow over an infinite rotating plate, indicates that thickness loses its memory of the initial profile with time, reaching a uniform distribution. Experimental procedures, as performed by Washo [3], also corroborate this fact, showing good reproducibility.

Today, polymers play an important role in the coating process due to their wide possibilities of application. Most polymers have a non-Newtonian behavior, and some of these obey the power-law model. Some numerical simulations, by Acrivos et al.

$1559-128 \mathrm{X} / 14 / 091820-06 \$ 15.00 / 0$

(C) 2014 Optical Society of America
[4] and Jenekhe and Schuldt [5], have shown that the flow becomes memoryless of the initial thickness profile, but without reaching uniformity.

Charpin et al. [6] and, more recently, Temple-Boyer et al. [7] presented an exact solution for the thickness profile of power-law fluids during spin coating. Their solution predicts a divergence in the spatial coordinate that forbids uniformity in the center of the plate.

On other hand, interferometric optical monitoring has risen as a powerful and accurate method for real-time and in situ thickness monitoring in dipcoating [8-10] and spin-coating [11-14] processes. In fact, the interferometric monitoring method is able to provide the typical parameters of the power-law fluid [15] with good accuracy and reproducibility. Moreover, the good agreement between the theoretical model and the experimental data corroborates the valid employment of the model, which allows neglecting the divergence and predicting the film uniformity, as expected.

In this work, a distinct analytical solution for non-Newtonian power-law fluids is shown in detail. 
This solution leads to the definition of the characteristic time that is associated with the process reproducibility. Reproducibility of a real process is investigated and model predictions are compared with experimental data from carboxymetilcellulose (CMC) under spin coating.

\section{Theory}

Consider a constant pressure, axisymmetric, steady state flux of a power-law fluid over an infinite rotating plate. In the disk, the equation of motion in cylindrical coordinates with radial direction $r$ [6] is

$$
\frac{\partial \tau_{r z}}{\partial z}=-\rho r \omega^{2},
$$

where $\omega$ is the rotation speed, $\tau_{r z}$ is a component of the stress tensor, and $\rho$ is the fluid density. The $z$ axis is perpendicular to the plate that is in the $z=0$ position. The free surface is at $z=h(r, t)$, where $t$ denotes time. The viscous shear stress balances the centrifugal force.

The boundary conditions

$$
v_{r}(z=0)=0
$$

and

$$
\tau_{r z}(z=h)=0
$$

represent the zero velocity $\nu_{r}(z)$ of the fluid on the disk surface [Eq. (2)] and the absence of stress $\tau_{r z}(z)$ in the fluid-air interface [Eq. (3)].

For a fluid that follows the power-law model, the stress tensor takes the form

$$
\tau_{r z}=K\left|\frac{\partial v_{r}}{\partial z}\right|^{l-1} \frac{\partial v_{r}}{\partial z} .
$$

The constant $l$ represents the power in the model. The $s$ values for the pseudoplastic case are in the range of $0<l<1$. The case $l>1$ is called dilatant. The Newtonian case is reached for $l=1$, and this is valid for any equation in this paper. $K$ is the rheological constant, associated with the effective viscosity defined by the equation

$$
\mu_{r z}^{\mathrm{eff}}=K\left|\frac{\partial v_{r}}{\partial z}\right|^{l-1},
$$

where $K$ becomes the fluid viscosity for $l=1$.

The velocity in the $\boldsymbol{r}$ direction, by substituting Eq. (ㅁ) in Eq. (1) and using boundary condition (3), can be written as

$$
v_{r}(z)=\left(\frac{\rho}{K} \omega^{2} r\right)^{\frac{1}{l}}\left(\frac{l}{l+1}\right)\left(h^{\frac{l+1}{l}}-(h-z)^{\frac{l+1}{l}}\right) .
$$

The flux $q$ for a circumference element,

$$
q=\int_{0}^{h} v_{r}\left(z^{\prime}\right) \mathrm{d} z^{\prime}
$$

by using Eq. ( $\underline{6})$ and boundary condition (2 $)$, becomes

$$
q=\frac{l}{2 l+1}\left(\frac{\rho}{K} \omega^{2} r\right)^{\frac{1}{l}} h^{\frac{2 l+1}{l}} .
$$

The flux ( ) is substituted into the continuity equation

$$
\frac{\partial h(r, t)}{\partial t}+\frac{1}{r} \frac{\partial(r q)}{\partial r}=0
$$

and gives the partial differential equation for the thickness:

$$
\frac{\partial h}{\partial t}+\frac{(l+1)}{(2 l+1)} h^{\frac{2 l+1}{l}}\left(\frac{\rho \omega^{2}}{K}\right)^{\frac{1}{l}} r^{\frac{1-l}{l}}+\left(\frac{\rho \omega^{2}}{K}\right)^{\frac{1}{l}} r^{\frac{1}{l}} h^{\frac{l+1}{l}} \frac{\partial h}{\partial r}=0 .
$$

This equation is separable, so its solution can be written as

$$
h(r, t)=R(r) T(t)
$$

and, using $\sigma$ as a separation constant, the spatial and temporal equations take, respectively, the form

$$
\frac{(l+1)}{(2 l+1)} R^{\frac{l+1}{l}} r^{\frac{1-l}{l}}+r^{\frac{1}{t}} R^{\frac{1}{l}} \frac{d R}{d r}-\sigma=0
$$

and

$$
\frac{1}{T^{2 l+1}} \frac{d T}{d t}=-\sigma\left(\frac{\rho \omega^{2}}{K}\right)^{\frac{1}{l}} .
$$

The temporal equation is easily integrated to

$$
T(t)=\left[T_{0}^{-\frac{(l+1)}{l}}+\frac{l+1}{l} \sigma\left(\frac{\rho \omega^{2}}{K}\right)^{\frac{1}{\tau}} t\right]^{-\frac{l}{l+1}},
$$

where $T_{o}$ represents the contribution at $t=0$. If $l=1$, then it represents the initial thickness for a given position.

The spatial Eq. (12) is an inexact ODE, and the integrand factor can be found. However, we prefer solving it in a distinct way, through a dimensional analysis, which may be useful for nonlinear differential equations without exact solution.

Now, a dimensional analysis is performed in order to inspire the Ansatz for the spatial solution. Considering $\omega=1 /[\mathrm{s}], K=[\mathrm{N}][\mathrm{s}]^{l} /[\mathrm{m}]^{2}$ and $r=[\mathrm{Kg}] /[\mathrm{m}]^{3}$, the temporal solution takes the dimension $T=$ $[\mathrm{m}]^{2 /(l+1)}$. As the dimension of the thickness must be $h=[\mathrm{m}]$, the spatial solution has the dimension $R=[\mathrm{m}]^{(l-1) /(l+1)}$ according to Eq. (11).

The Ansatz for the spatial solution can be presented as 


$$
R(r)=\operatorname{Cr}^{\frac{(l-1)}{(l+1)}}
$$

The initial thickness profile $h_{0}$ is defined for an arbitrary position $r_{0}$, so that, by combining Eqs. (14) and (15) at $t=0$,

$$
C=h_{0}\left[r_{0}^{\frac{(l-1)}{(l+1)}} T_{0}\right]^{-1}
$$

The total solution for thickness evolution at arbitrary $t$ and $r$,

$$
h(r, t)=h_{0}\left(\frac{r}{r_{0}}\right)^{\frac{(l-1)}{l+1)}}\left[1+\frac{(l+1)}{l} \sigma T_{0}^{\frac{(l+1)}{l}}\left(\frac{\rho \omega^{2}}{K}\right)^{\frac{1}{\tau}} t\right]^{-\frac{l}{(l+1)}},
$$

predicts a divergence at $r=0$, which can be attributed to the simplicity of the model, without explicit consideration of both surface tension and gravity, which leads to the divergence of the viscosity in that point. Fortunately the nonuniformity area around $r=0$ is very small and an experimental probe area can be founded very near the center of the plate.

To determine the value of the separation constant $\sigma$, the total solution (17) must satisfy the differential Eq. (10), so that

$$
\sigma T_{0}^{\frac{(l+1)}{l}}=h_{0}^{\frac{(l+1)}{l}} r_{0}^{\frac{(1-l)}{l}} \frac{(3 l+1) l}{(2 l+1)(l+1)} .
$$

The solution of the partial differential Eq. (10) then becomes

$$
h(r, t)=\left(\frac{r}{r_{0}}\right)^{\frac{(l-1)}{l+1)}}\left[h_{0}^{\frac{-(l+1)}{l}}+\frac{(3 l+1)}{(2 l+1)} r_{0}^{\frac{(1-l)}{l}}\left(\frac{\rho \omega^{2}}{K}\right)^{\frac{1}{l}} t\right]^{-\frac{l}{(l+1)}}
$$

This is exactly the previously reported solution [7]. In our case, the dimensional analysis arises as a powerful instrument that leads to the solution.

For large times, for which

$$
t \gg t_{c},
$$

where the characteristic time is defined as

$$
t_{c} \equiv \frac{(2 l+1)}{(3 l+1)} \frac{r_{0}^{\frac{(l-1)}{l}}}{h_{0}^{\frac{(l+1)}{l}}}\left(\frac{K}{\rho \omega^{2}}\right)^{\frac{1}{l}}
$$

the thickness evolution loses dependence on its initial value, as experimentally expected from the observed process reproducibility, and starts to depend strongly on the rotation speed, power, and rheological constant. For $l=1$, the Newtonian case is recovered and the thickness no longer depends directly on the spatial position, reaching a uniform profile after the stationary regime is attained.
As the power in the Newtonian case $(l=1)$ is larger than that for the pseudoplastic case $(0<l<1)$, for the same evolution time, the thickness for the latter is smaller than that for the former.

\section{Experimental}

Optospinigraphy [11] allows real-time thickness monitoring through successive reflectance extrema, whose distance is associated with a quarter of a wavelength. Light from a laser diode $\left(\lambda_{o}=660 \mathrm{~nm}\right)$ is used in the interferometer, which is coupled to the spinner and generates for the reflectance, $R$, a sinusoidal profile as the fluid film flows outward in the disk, in accordance with

$$
R=R(\cos \delta),
$$

where $\delta$ is the phase variation of the reflected light. More detailed equipment description and experimental analysis are reported in Ref. [15].

The reflectance signal of a coherent light, at normal incidence in a fixed position $r$, changes its phase $\delta$ with optical path, during flow, as

$$
\delta=\frac{4 \pi}{\lambda_{0}} n_{1} h,
$$

where $n_{1}$ is the film refractive index and $h$ is its thickness.

In ideal conditions, the reflectance amplitude is reproduced for $\delta=\delta+2 \pi$, or for a physical thickness variation $h \rightarrow h+\Delta h$. The optical thickness variation reaches an extremum when

$$
\Delta h n_{1}=m \frac{\lambda_{0}}{4}, \quad \text { where } m=1,2,3, \ldots
$$

The thickness variation in time is associated with the occurrence of extrema in the reflectance signal and is proportional to integer multiples of a quarter of the wavelength.

The CMC polymer was chosen due to its typical power-law behavior, previously verified [10], and to its synthesis simplicity. The refraction indices for distinct weight concentrations in water were obtained by use of an Abbe refractometer (see Table 1), and these concentrations were tested under different rotation speeds at controlled temperature $\left(\right.$ Temp $\left.=22.4{ }^{\circ} \mathrm{C}\right)$ and humidity (Humid $\left.=100 \%\right)$.

The reflectance output, exemplified in Fig. 1, for distinct concentrations at a fixed rotation speed [15], exhibits a sinusoidal-like profile, as expected. The physical thicknesses variations are shown in Fig. 2 .

Table 1. Refraction Indices, $n_{1}$, for CMC Weight Concentrations, Measured by an Abbe Refractometer

\begin{tabular}{lc}
\hline Concentration in Water $(\%$ wt. $)$ & $n_{1}$ \\
\hline 0.1 & $1.3332 \pm 0.0005$ \\
0.2 & $1.3333 \pm 0.0005$ \\
0.5 & $1.3335 \pm 0.0005$ \\
\hline
\end{tabular}



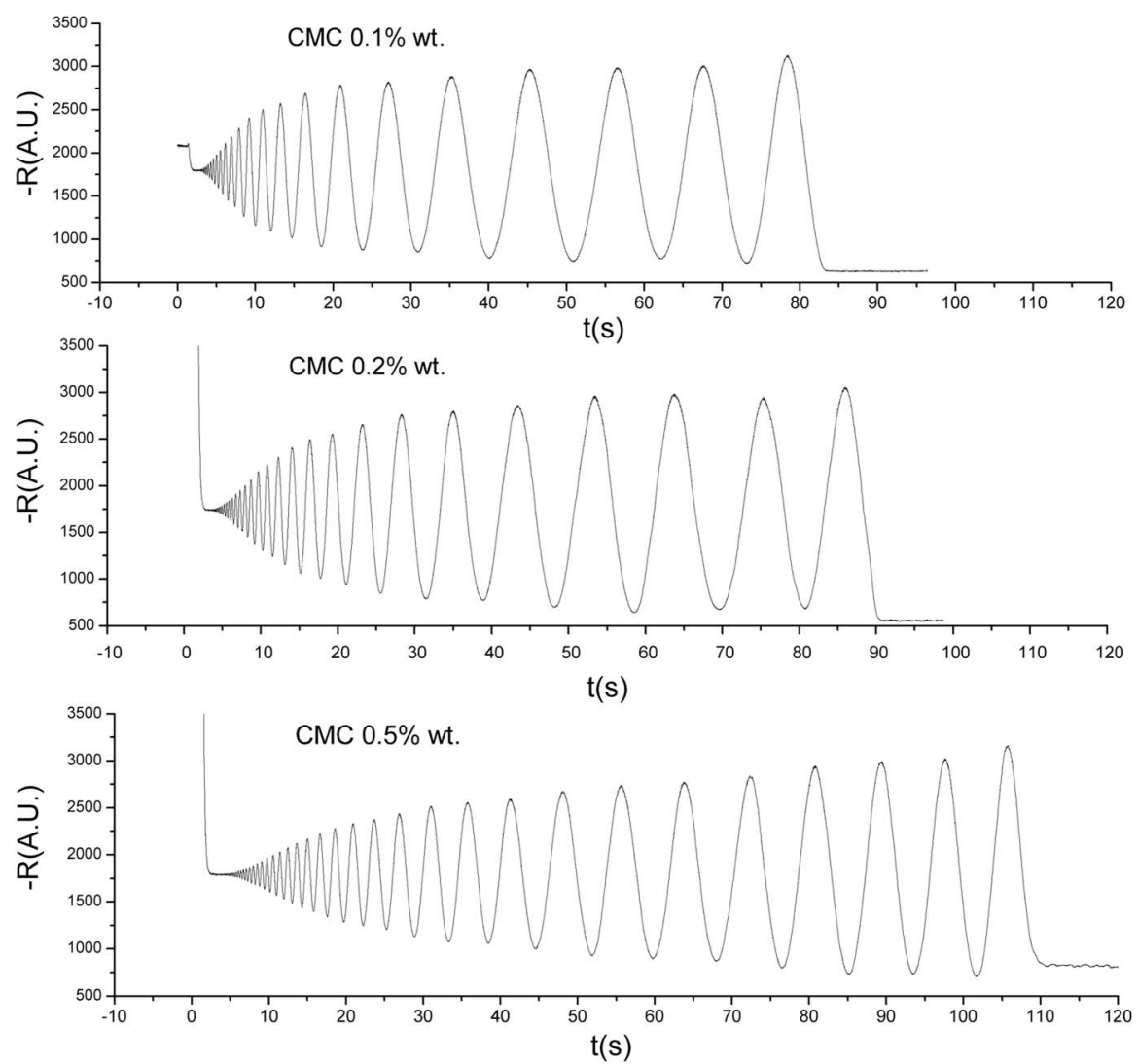

Fig. 1. Reflectance profile for $0.1 \%$ wt., $0.2 \%$ wt., and $0.5 \%$ wt. CMC at a rotation speed of $5000 \mathrm{rpm}$. (Reflectance signal is inverted due to lock-in amplifier response polarity).

Each point represents the occurrence of an extremum in the reflectance signal.

The proposed solution for power-law fluids, Eq. (19), fits well with the experimental data, Fig. 2, considering a negative thickness variation $\Delta h=h_{0}-h$ and a measuring position, $r=r_{0}$, nearly the center. This allows an accurate evaluation of the flow parameters $l$ and $K$ (see Table 2). These parameters can also be obtained for other controlled rotation speeds [15], as in Table $\underline{2}$.

\section{Discussion}

The characteristic times, calculated from its definition, Eq. (21), lie in the range of 0.5-1.4 s; see Table 2. The total times of the flows over the disk, $t_{t}$, for the measured set of CMC concentrations and rotation speeds, are approximately two orders of magnitude larger than the characteristic times; see Table 2. This indicates the precise evaluation of the power $l$ and of the rheological constant $K$, according to the condition (20). The reproducibility in parameter evaluation can be observed in Table 2 , where the values of $K$ are nearly the same for a given CMC concentration at different rotation speeds.

As Eq. (24) depends on the thickness variation $\Delta h=h_{o}-h$, for times of the order of the characteristic time $t_{c}$, thickness variation profiles, as in Fig. 2 , are superimposed, indicating the dominance of dependency on initial thickness.
With increasing time, these profiles split and evolve separately. This suggests that the dependence of variation thickness on the rheological constant, power, and rotation speed becomes increasingly stronger, as can be seen through Eq. (19). The existence of this region for a sufficient time period leads to a precise determination of the power $l$ and the rheological parameter $K$, and this time period depends on characteristic time value.

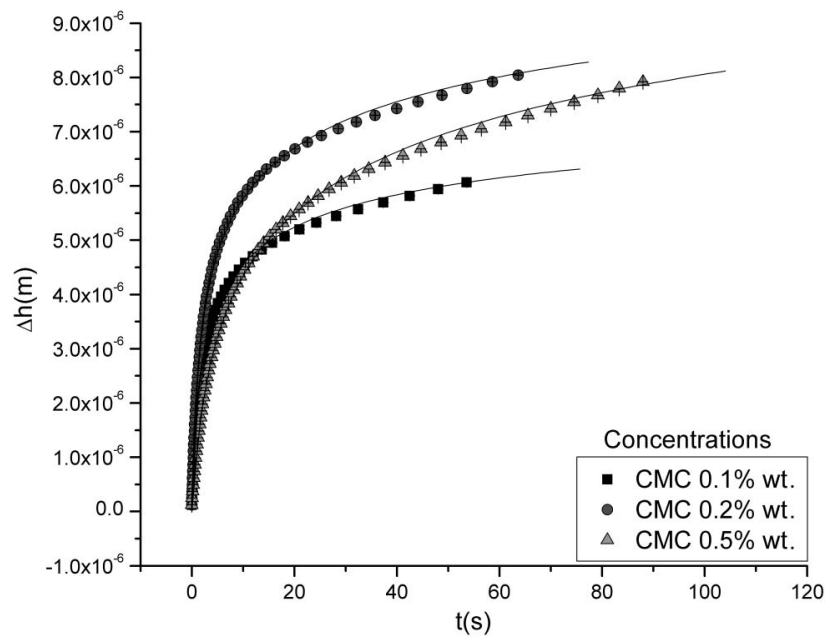

Fig. 2. Physical thickness variation for $0.1 \%$ wt., $0.2 \%$ wt., and $0.5 \%$ wt. CMC at a rotation speed of $5000 \mathrm{rpm}$. 
Table 2. Rheological Constants $(K)$, Powers $(I)$, Characteristic Times $\left(t_{c}\right)$, Total Flow Times $\left(t_{t}\right)$, and Initial Thicknesses in the Steady State $\left(h_{o}\right)$ for $0.1 \%$ wt., $0.2 \%$ wt., and $0.5 \%$ wt. CMC at Several Rotation Speeds $\omega$

\begin{tabular}{|c|c|c|c|c|c|c|}
\hline Concentration in Water (\% wt.) & $\omega(\mathrm{rpm})$ & $h_{o}(\mu \mathrm{m})$ & $l$ & $K\left(\mathrm{~Pa} \cdot \mathrm{s}^{l}\right)$ & $t_{c}(s)$ & $t_{t}(s)$ \\
\hline 0.1 & 2500 & $16.6 \pm 0.7$ & $0.18 \pm 0.02$ & $0.0077 \pm 0.0007$ & 0.81 & 124.71 \\
\hline 0.1 & 3000 & $13.7 \pm 0.5$ & $0.23 \pm 0.02$ & $0.0077 \pm 0.0007$ & 0.97 & 91.22 \\
\hline 0.1 & 3500 & $12.1 \pm 0.4$ & $0.26 \pm 0.02$ & $0.0077 \pm 0.0008$ & 0.8 & 77.08 \\
\hline 0.1 & 4000 & $10.9 \pm 0.3$ & $0.28 \pm 0.02$ & $0.0078 \pm 0.0009$ & 0.68 & 59.79 \\
\hline 0.1 & 5000 & $9.3 \pm 0.4$ & $0.30 \pm 0.03$ & $0.009 \pm 0.001$ & 0.64 & 59.49 \\
\hline 0.2 & 2500 & $33 \pm 2$ & $0.083 \pm 0.007$ & $0.023 \pm 0.002$ & 1.00 & 141.67 \\
\hline 0.2 & 3000 & $27 \pm 2$ & $0.11 \pm 0.01$ & $0.023 \pm 0.003$ & 0.52 & 113.30 \\
\hline 0.2 & 3500 & $21 \pm 1$ & $0.14 \pm 0.01$ & $0.022 \pm 0.002$ & 0.67 & 92.23 \\
\hline 0.2 & 4000 & $17 \pm 1$ & $0.15 \pm 0.02$ & $0.023 \pm 0.003$ & 0.97 & 80.13 \\
\hline 0.2 & 5000 & $14.5 \pm 0.6$ & $0.21 \pm 0.02$ & $0.022 \pm 0.003$ & 0.58 & 71.75 \\
\hline 0.5 & 4000 & $36 \pm 3$ & $0.087 \pm 0.008$ & $0.063 \pm 0.006$ & 0.83 & 117.49 \\
\hline 0.5 & 4500 & $29 \pm 2$ & $0.094 \pm 0.008$ & $0.065 \pm 0.006$ & 1.43 & 101.63 \\
\hline 0.5 & 5000 & $24 \pm 2$ & $0.10 \pm 0.01$ & $0.064 \pm 0.007$ & 1.37 & 88.34 \\
\hline
\end{tabular}

Thickness variations that are approximately superimposed must have near values for the characteristic time, because they have approximate flow parameters. In this sense, considering distinct thickness variation profiles, the greatest superimposition during the initial times represents the nearest values for the characteristic times. This tendency can be seen in Fig. 2. Thickness profiles for concentrations of CMC $0.1 \%$ wt. and $0.2 \%$ wt., at $5000 \mathrm{rpm}$, have greater superimposition in initial times than the CMC $0.5 \%$ wt. profile. The characteristic times for the lower CMC concentrations are smaller and closer than the characteristic time of the CMC $0.5 \%$ wt. (see Table 2).

According to Eq. (21), the characteristic time is inversely proportional to the initial thickness. The initial thickness is associated with the detection of the first extremum of the reflectance after steady state flow is reached. Therefore, a sooner detection of the first extremum benefits the precision and the reproducibility of the evaluation of the flow parameters. The detection of the first extremum is related to factors such as the acquisition rate of the light detector, the absorption of light by the fluid, and when the steady state flow is reached. The low characteristic times in comparison with the total flow time attest to the efficiency of the measure device and the measurement quality in the determination of flow parameters.

\section{Conclusions}

The exact analytical solution to non-Newtonian power-law fluids, under spin coating whose distinct development was hereby presented, has provided a good fitting with the experimental data, leading to accurate parametric evaluation. Moreover, the solution allows the definition of a characteristic time, which is related to the thickness temporal profile and process reproducibility.

The thickness-variation profiles and the characteristic times were obtained from experimental data, using different concentrations of CMC. The low values obtained for the characteristic times attest to the process reproducibility, as observed experimentally.
Similarly to that observed in the Newtonian case $[12,13]$, for times much larger than $t_{c}$, the powerlaw process becomes memoryless of initial thickness.

The good fitting attained (Fig. 2), under several rotation speeds and concentrations, in Table 2 indicates the consistency of the theoretical approach. The small values obtained for the characteristic time, as in Table 2, corroborate the rheological precision of the measurement process after steady state conditions are reached.

This work was supported by Coordenação de Aperfeiçoamento de Pessoal de Nível Superior (CAPES) and by Conselho Nacional de Desenvolvimento Científico e Tecnológico (CNPq). Special thanks are due to Eng. Roberto Spinato for technical support in the Laser and Film Optics Laboratory at UFRGS.

\section{References}

1. P. H. Walker and J. G. Thompson, "Proceedings of the twentyfifth annual meeting," Am. Soc. Test. Mater. 22, 463-485 (1922).

2. A. G. Emslie, F. T. Bonner, and L. G. Peck, "Flow of a viscous liquid on a rotating disk," J. Appl. Phys. 29, 858-862 (1958)

3. B. D. Washo, "Rheology and modeling of the spin coating process," IBM J. Res Devel. 21, 190-198 (1977).

4. A. Acrivos, M. J. Shah, and E. E. Petersen, "On the flow of a non-Newtonian liquid on a rotating disk," J. Appl. Phys. 31, 963-968 (1960)

5. S. A. Jenekhe and S. B. Schuldt, "Coating of non-Newtonian fluids on a flat rotating disk," Ind. Eng. Chem. Fundam. 23, 432-436 (1984).

6. J. P. F. Charpin, M. Lombe, and T. G. Myers, "Spin coating on non-Newtonian fluids with a moving front," Phys. Rev. E 76 0163121 (2007).

7. P. Temple-Boyer, L. Mazenq, J. B. Doucet, V. Conédéra, B. Torbiéro, and J. Launay, "Theoretical studies of the spin coating process for the deposition of polymer-based Maxwellian liquids," Microelectron. Eng. 87, 163-166 (2010).

8. A. F. Michels, T. Menegotto, and F. Horowitz, "Optically monitored dip coating as a contactless viscometry method for liquid films," Appl. Opt. 44, 912-915 (2005).

9. A. F. Michels, T. Menegotto, F. Horowitz, M. B. Susin, and H. P. Grieneisen, "Double optical monitoring of dip coating with a time-varying refractive index," Appl. Opt. $\mathbf{4 5}$, 1491-1494 (2006).

10. A. F. Michels, P. L. G. Jardim, and F. Horowitz, "Laser monitoring of non-Newtonian liquids during dip coating," AIChe J. 55, 3052-3055 (2009). 
11. F. Horowitz, E. Yeatman, E. Dawnay, and A. Fardad, "Realtime optical monitoring of spin coating," J. Phys. III France 3, 2059-2063 (1993).

12. F. Horowitz, E. Yeatman, E. Dawnay, and A. Fardad, "Optical monitoring of the sol-to gel transition in spinning silica films," Proc. SPIE 2288, 67-70 (1994).

13. F. Horowitz, E. J. C. Dawnay, M. A. Fardad, M. Green, and E. M. Yeatman, "Towards better control of sol-gel film processing for optical device applications," J. Nonlinear Opt. Phys. Mater. 6, 1-18 (1997).

14. F. Horowitz, A. F. Michels, and E. M. Yeatman, "Optical viscometry of spinning sol coatings," J. Sol-Gel Sci. Technol. 13, 707-712 (1998).

15. P. L. G. Jardim, A. F. Michels, and F. Horowitz, "Optical interference monitoring for power-law fluids during spin coating," Opt. Express 20, 3166-3175 (2012). 